\title{
Multiprotein Complexes of the Survival of Motor Neuron Protein SMN with Gemins Traffic to Neuronal Processes and Growth Cones of Motor Neurons
}

\author{
Honglai Zhang, ${ }^{2 \star}$ Lei Xing, ${ }^{1 \star}$ Wilfried Rossoll, ${ }^{1}$ Hynek Wichterle, ${ }^{3}$ Robert H. Singer, ${ }^{2}$ and Gary J. Bassell ${ }^{1}$ \\ ${ }^{1}$ Department of Cell Biology and Center for Neurodegenerative Disease, Emory University, Atlanta, Georgia 30322, ${ }^{2}$ Department of Anatomy and Structural \\ Biology, Albert Einstein College of Medicine, Bronx, New York 10461, and ${ }^{3}$ Department of Pathology, Columbia University College of Medicine, New York, \\ New York 10032
}

\begin{abstract}
Spinal muscular atrophy (SMA), a progressive neurodegenerative disease affecting motor neurons, is caused by mutations or deletions of the SMN1 gene encoding the survival of motor neuron (SMN) protein. In immortalized non-neuronal cell lines, SMN has been shown to form a ribonucleoprotein (RNP) complex with Gemin proteins, which is essential for the assembly of small nuclear RNPs (snRNPs). An additional function of SMN in neurons has been hypothesized to facilitate assembly of localized messenger RNP complexes. We have shown that SMN is localized in granules that are actively transported into neuronal processes and growth cones. In cultured motor neurons, SMN granules colocalized with ribonucleoprotein Gemin proteins but not spliceosomal Sm proteins needed for snRNP assembly. Quantitative analysis of endogenous protein colocalization in growth cones after three-dimensional reconstructions revealed a statistically nonrandom association of SMN with Gemin2 (40\%) and Gemin3 (48\%). SMN and Gemin containing granules distributed to both axons and dendrites of differentiated motor neurons. A direct interaction between SMN and Gemin2 within single granules was indicated by fluorescence resonance energy transfer analysis of fluorescently tagged and overexpressed proteins. High-speed dualchannel imaging of live neurons depicted the rapid and bidirectional transport of the SMN-Gemin complex. The N terminus of SMN was required for the recruitment of Gemin2 into cytoplasmic granules and enhanced Gemin2 stability. These findings provide new insight into the molecular composition of distinct SMN multiprotein complexes in neurons and motivation to investigate deficiencies of localized RNPs in SMA.
\end{abstract}

Key words: survival of motor neuron protein; SMN; spinal muscular atrophy; SMA; RNA granules; mRNA localization; growth cone; motor neuron disease

\section{Introduction}

Spinal muscular atrophy (SMA) is a common inherited disease, characterized by neurodegeneration of $\alpha$-motor neurons (Frugier et al., 2002). SMA is caused by mutation and/or deletion of the SMN1 gene that encodes the survival of motor neuron protein (SMN) (Frugier et al., 2002). SMN localizes to both the cytoplasm and the nucleus; in the nucleus, it is found in gems (Liu and Dreyfuss, 1996) that often colocalize with coiled (Cajal) bodies (Carvalho et al., 1999; Young et al., 2000). Based on biochemistry studies of non-neuronal cell lines, several SMN-associated proteins, named Gemins, have been identified as integral components of the SMN ribonucleoprotein complex (Gubitz et al., 2004; Yong et al., 2004). Although it is clear that a critical function

Received Sept. 19, 2005; revised July 10, 2006; accepted July 10, 2006.

This work was supported by grants from the Spinal Muscular Atrophy Foundation, the Ritter Foundation, the Muscular Dystrophy Association, and Families of SMA. We thank Mike Lorenz, Shailesh Shenoy, and Wulin Teo for assistance with microscopy and image analysis. We thank Utz Fischer and Gideon Dreyfuss for providing antibodies. ${ }^{*} H . Z$. and L.X. contributed equally to this work.

Correspondence should be addressed to Dr. Gary Bassell, Department of Cell Biology, Emory University, 615 Michael Street, Atlanta, GA 30322. E-mail: gbassel@emory.edu.

DOI:10.1523/JNEUROSCI.3967-05.2006

Copyright $\odot 2006$ Society for Neuroscience $\quad$ 0270-6474/06/268622-11\$15.00/0 for the SMN-Gemin complex is to act as an assembly machine to facilitate spliceosomal small nuclear ribonucleoprotein (snRNP) assembly (Paushkin et al., 2002), it is unclear why motor neurons are more vulnerable to loss of SMN. One hypothesis is that reduction of SMN levels in neurons (particularly motor neurons) may compromise splicing machinery more seriously (Gubitz et al., 2004). So far, there is no evidence for mRNA splicing defects in SMA patients or SMA models.

An alternative view is that the SMN complex is used for an additional function in neurons, such as assembly and regulation of localized messenger RNP (mRNP) complexes (for review, see Briese et al., 2005; Monani, 2005). Previous immunocytochemical studies have detected SMN in both dendrites and axons in vivo (Bechade et al., 1999; Pagliardini et al., 2000). Using immunofluorescence on cultured primary neurons, endogenous SMN was localized in granules that extend throughout processes and into growth cones (Zhang et al., 2003). Live cell imaging has revealed rapid and cytoskeletal-dependent movements of enhanced green fluorescent protein (EGFP)-SMN granules (Zhang et al., 2003). SMN was shown to bind heterogeneous nuclear ribonucleoprotein-R (hnRNP-R) (Rossoll et al., 2002), an mRNA binding protein that can associate with $\beta$-actin mRNA in vitro (Rossoll et 
al., 2003). Although the precise role of hnRNP-R in the mechanism of $\beta$-actin mRNA localization is unknown, deficiency of SMN, in a mouse transgenic model of SMA (Monani et al., 2000), results in reduced localization of $\beta$-actin mRNA in cultured motor neurons (Rossoll et al., 2003). This study suggests some role of $\mathrm{SMN}$ in the assembly and/or localization of $\beta$-actin mRNP complexes. Because previous studies have identified an important function for $\beta$-actin mRNA localization (Zhang et al., 2001) and local protein synthesis (Campbell and Holt, 2001) in mediating growth cone motility and chemotropic responses, the hypothesis that SMN participates in some aspect of cytoplasmic-directed RNA localization and/or translation in growth cones is very appealing.

To understand how SMN may be involved in the assembly and/or localization of ribonucleoprotein complexes in neurons, it is first necessary to identify the protein components of SMN granules and elucidate their molecular interactions with SMN. Past studies have been inconclusive on whether Gemin proteins are present as particles within neuronal processes and whether they colocalize with SMN. In one report, SMN was distributed in a particulate pattern in processes of cultured motor neurons, yet Sip1 (Gemin2) was uniformly distributed and showed little colocalization (Jablonka et al., 2001). More recently, SMN and several of the Gemin proteins were shown by double-labeled immunofluorescence (IF) to be coenriched in neuritic protrusions of PC12 cells (Sharma et al., 2005), however, this study did not use methods to permit assessment of whether SMN and Gemins colocalize within individual particles or granules. In this study, we used fluorescence microscopy, digital imaging, and quantitative analyses of live and fixed neurons to demonstrate the formation of an SMN-Gemin complex in neuritic granules, yet our data also suggest the presence of SMN granules lacking Gemins. In addition, we show that molecular interactions between SMN and Gemin proteins can affect the assembly and localization of this complex.

\section{Materials and Methods}

Chick forebrain and rat hippocampal cultures. Chick forebrain neurons were cultured as described previously (Zhang et al., 2001, 2003). Briefly, chick forebrains [embryonic day 8 (E8)] were dissected, trypsinized $(0.15 \%$ in $\mathrm{HBSS})$ at $37^{\circ} \mathrm{C}$ for $7 \mathrm{~min}$, and plated on poly-L-lysine $(0.4$ $\mathrm{mg} / \mathrm{ml})$ - and laminin $(0.02 \mathrm{mg} / \mathrm{ml})$-coated coverslips. Cells were inverted onto a monolayer of astrocytes in $\mathrm{N}_{3}$-conditioned medium with $2 \% \mathrm{FBS}$ and cultured for $4 \mathrm{~d}$ at $37^{\circ} \mathrm{C}$ in $5 \% \mathrm{CO}_{2} \cdot \mathrm{N}_{3}$-conditioned medium containing Eagle's minimum essential medium supplemented with transferrin $(0.2 \%)$, ovalbumin $(0.1 \%)$, insulin $(10 \mu \mathrm{g} / \mathrm{ml})$, putrescine (32 $\mu \mathrm{g} / \mathrm{ml})$, sodium selenite $(26 \mathrm{ng} / \mathrm{ml})$, progesterone $(12.5 \mathrm{ng} / \mathrm{ml})$, hydrocortisone $(9.1 \mathrm{ng} / \mathrm{ml})$, T3 (3,3',5'-triiodo-L-thyronine, sodium salt, $20 \mathrm{ng} / \mathrm{ml})$, and BSA (10 $\mu \mathrm{g} / \mathrm{ml})$. Rat hippocampi (E18) were cultured for $4 \mathrm{~d}$ as described previously (Antar et al., 2004).

Primary and embryonic stem cell-derived motor neuron cultures. Primary mouse motor neuron cultures were prepared from E13.5 mouse spinal cords essentially as described previously (Arce et al., 1999), but the magnetic column step was omitted, and metrizamide was replaced by Optiprep (10\%; Nycomed Pharma, Oslo, Norway) for gradient centrifugation. Cells in the motor neuron-enriched fraction were plated on 15 $\mathrm{mm}$ glass coverslips coated with poly-ornithine/laminin. Motor neurons were identified by morphological criteria and by immunofluorescence staining of choline acetyltransferase (ChAT) with rabbit anti-ChAT (Chemicon, Temecula, CA). More than $90 \%$ of the purified cells were positive for ChAT (data not shown). Culture medium was Neurobasal (Invitrogen, Carlsbad, CA) supplemented with B27 (Invitrogen), horse serum ( $2 \% \mathrm{v} / \mathrm{v}$; Sigma, St. Louis, MO), Glutamax-1 (0.5 mM; Invitrogen), 2-mercaptoethanol $(25 \mu \mathrm{M})$, and BDNF and CNTF (10 ng/ml; PeproTech, Rocky Hill, NJ).
Mouse HB9::EGFP embryonic stem (ES) cells (HBG3 cells) were cultured and differentiated into motor neurons as described previously (Wichterle et al., 2002) with the following minor modifications. ES cells were plated at $10^{5}$ cells/ml in DFNK medium [DMEM/F-12/Neurobasal in 1:1:2 ratio supplemented with 10\% knock-out Serum Replacement (Invitrogen), $0.1 \mathrm{~mm}$ 2-mercaptoethanol (Sigma), $200 \mathrm{~mm} \mathrm{L-glutamine,}$ and $1 \times$ penicillin/streptomycin (Invitrogen when not specified)]. Twoday-old embryoid bodies were induced with $1 \mu \mathrm{M}$ retinoic acid (Sigma) and $1 \mu \mathrm{M}$ HhAg1.3 (Frank-Kamenetsky et al., 2002). Four days after induction, motor neurons were dissociated using the papain dissociation system (Worthington, Freehold, NJ), plated on poly-D-lysine and lami$\operatorname{nin}(20 \mu \mathrm{g} / \mathrm{ml})$-coated coverslips, cultured for $3 \mathrm{~d}$, and fixed.

Immunofluorescence. All cells were fixed in paraformaldehyde (4\% in $1 \times \mathrm{PBS}$ ) for $20 \mathrm{~min}$ at room temperature and washed in $1 \times \mathrm{PBS}$ with 5 $\mathrm{mm} \mathrm{MgCl}_{2}$ three times.

Monoclonal antibodies to SMN (1:1000; BD Biosciences, San Jose, CA), Gemin3 (1:1000; Abcam, Cambridge, UK), and a rabbit antibody to Gemin2 (1:1000; provided by Utz Fischer, University of Wuerzburg, Wuerzburg, Germany) (Jablonka et al., 2001) were used for detection of the endogenous proteins in cultured chick forebrain and ES cell-derived motor neurons. To analyze the distribution of snRNPs with respect to SMN, primary cultures of motor neurons were double stained with a monoclonal antibody (Y12) to spliceosomal Sm proteins (1:2000; provided by Utz Fischer) and a rabbit polyclonal antibody to SMN (1:500; Santa Cruz Biotechnology, Santa Cruz, CA), the specificity of which was tested by Western blot. Primary antibodies were detected by affinitypurified donkey antibodies to mouse or rabbit IgG conjugated to fluorochromes, cyanine 3 (Cy3) or Cy5 (Jackson ImmunoResearch, West Grove, PA). For quantitative colocalization analysis, mouse anti-SMN was conjugated with Alexa Fluor 647, and mouse anti-Gemin2, antiGemin3, and anti-synaptophysin (Sigma) were conjugated with Alexa Fluor 568 using Zenon mouse IgG1 labeling kits (Invitrogen) according to the instructions of the manufacturer. Alexa fluorochrome-conjugated antibodies were used at 1:600 for mouse anti-SMN, anti-Gemin2, and anti-Gemin 3 antibodies and at 1:200 for mouse anti-synaptophysin. Alexa fluorochrome-conjugated mouse antibodies to SMN and Gemin2 were also used to detect SMN-Gemin complex in primary cultures of rat hippocampal neurons and mouse motor neurons. Axons and dendrites were discriminated by IF with rabbit anti-tau (1:2000; Sigma) or rabbit anti-microtubule-associated protein 2 (MAP2) (1:1500; Sigma) and visualized with Cy2-conjugated secondary antibody (Jackson ImmunoResearch). Antibody incubations were for $1 \mathrm{~h}$ at room temperature in Trisbuffered saline (TBS) with BSA (2\%) and Triton X-100 (0.1\%). Coverslips were mounted in a mount medium, as described previously (Zhang et al., 2001).

Fluorescence protein-reporter constructs. Full-length cDNA of the human SMN1 was subcloned into pEGFP-C1 (EGFP-SMN) as described previously (Zhang et al., 2003). In the present study, full-length cDNA of SMN1 was inserted into phosphorylated enhanced yellow fluorescent protein (pEYFP)-C1 (BD Biosciences Clontech, Palo Alto, CA) using HindIII and EcoRI sites (YFP-SMN). Full-length cDNAs of human Gemin 2 and Gemin3 were obtained by reverse transcription-PCR from total RNA extracts of HEK293 cells. Gemin 2 was inserted into pEGFP-C1 or phosphorylated enhanced cyan fluorescent protein (pECFP)-C1 (BD Biosciences Clontech) using BglII and EcoRI sites (EGFP-Gemin2 or ECFP-Gemin2); Gemin3 was inserted into pEGFP-C1 or pECFP-C1 using BglII site (EGFP-Gemin3 or ECFP-Gemin3). To determine domaindependent interaction of SMN with Gemin2, a human SMN cDNA with deletions of exon- 1 and -exon2a (the first 53 amino acids), which includes the 39 amino acids (encoded by chick SMN cDNA) that contains the Gemin2 binding site (Wang and Dreyfuss, 2001b), was generated using PCR primers and then subcloned into PEYFP-C1 (EYFP-SMN $\Delta$ N53). For coimmunoprecipitation (co-IP) experiments, YFP sequence was substituted with a Flag tag (MDYKDDDDK) in the above SMN constructs using AgeI and HindIII sites (Flag-SMN, Flag-SMN $\Delta 7$, and Flag-SMN $\Delta$ N53). All of the constructs were purified (Qiagen, Hilden, Germany) and sequenced to ensure that no frame shift had occurred.

Transfection. Neuronal transfections were performed using 1,2dioleoyl-3-trimethylammonium-propane (DOTAP) liposomal reagent 
(Roche, Indianapolis, IN), as described previously (Zhang et al., 2001, 2003). In cotransfection experiments, two DNA constructs were mixed equivalently (2-3 $\mu \mathrm{g}$ in total), diluted to $100 \mu \mathrm{l}$ with transfection buffer (20 mm HEPES and $150 \mathrm{~mm} \mathrm{NaCl}, \mathrm{pH}$ 7.4) and then incubated with DOTAP $(5 \mu \mathrm{l})$, as described above.

HEK293 cells were transfected with Flag- and EGFP-tagged constructs. HEK293 cells were cultured in DMEM containing 10\% FBS (Sigma). Cells were briefly washed with prewarmed medium before transfection. Equivalent amount of Flag- and EGFP-tagged constructs (2-3 $\mu \mathrm{g}$ in total) were diluted to $100 \mu \mathrm{l}$ in the transfection buffer and then incubated with $5 \mu \mathrm{l}$ of FuGENE 6 transfection reagent (Roche) for 15 min at room temperature. After incubation with the DNA mixtures for $1 \mathrm{~h}$, the cells were cultured in DMEM containing 10\% FBS for $24 \mathrm{~h}$. Protein lysates were prepared as described below.

Fluorescence microscopy and digital imaging. Fixed neurons were visualized using a Nikon (Tokyo, Japan) Eclipse inverted microscope equipped with a $60 \times$ Plan-Neofluar objective, phase optics, $100 \mathrm{~W}$ mercury arc lamp, and HiQ bandpass filters (Chroma Technology, Brattleboro, VT). Images were captured with a cooled CCD camera (Quantix; PhotoMetrics, Huntington Beach, CA) using a $35 \mathrm{~mm}$ shutter and processed using IP Lab Spectrum (Scanalytics, Fairfax, VA). Fluorescence images were acquired with specific filters, including Cy2, Cy3, or Cy5. In the transfected neurons, specific signals of EGFP, ECFP, and EYFP were observed using narrow bandpass filters. Exposure time was kept constant and below grayscale saturation.

Live neurons that coexpressed ECFP-Gemin2 or ECFP-Gemin3 with EYFP-SMN were imaged in a sealed environmental chamber (Focht chamber; Bioptechs, Butler, PA) using a T.I.L.L. Photonics (Martinsried, Germany) Imaging System. ECFP and EYFP images were alternately acquired by a CCD camera (Imago QE; T.I.L.L. Photonics) and synchronized with a monochromator with millisecond response time that switched between $442 \mathrm{~nm}$ (ECFP) and $510 \mathrm{~nm}$ (EYFP) excitation (Polychrome II; T.I.L.L. Photonics). Images were captured at an exposure rate of $0.5 \mathrm{~s}$ for each frame, with a total of 200 frames.

Colocalization analysis. To quantify the colocalization of SMN and Gemin proteins, rat hippocampal neurons were double stained with Alexa Fluor 647-conjugated mouse anti-SMN and Alexa Fluor 568conjugated mouse anti-Gemin2 and anti-Gemin3, respectively, or to anti-synaptophysin, as a negative control. Growth cones were imaged in each channel along the $z$-axis (11 sections at $0.1 \mu \mathrm{m}$ each), and five in-focus sections from each $z$-stack were deconvolved using a threedimensional (3D) blind algorithm [Autoquant X (Media Cybernetics, Silver Spring, MD); Bitplane (St. Paul, MN)] and analyzed for colocalization (see below). Images were registered using fiduciary beads (conjugated with multiple fluorochromes) present in mounting medium.

Colocalization was measured using ImarisColoc (Imaris 4.5.2; Bitplane). The threshold of each channel used to quantify colocalization was determined by creating IsoSurface (Imaris 4.5.2; Bitplane), which represents the signal range of a dataset. Colocalization was defined as the overlap of two channels in three dimensions and was calculated by the program automatically. The degree of colocalization was represented by percentage of voxels of each channel above the threshold colocalized. Ten datasets from each sample were analyzed with an unpaired Student's $t$ test.

Fluorescence resonance energy transfer. To evaluate whether SMN interacts with Gemin proteins within granules, fluorescence resonance energy transfer (FRET) was performed on neurons that coexpressed EYFPSMN and either ECFP-Gemin2 or ECFP-Gemin3. ECFP as a donor and EYFP as an acceptor is a preferred pair for FRET analysis because the emission spectrum of ECFP significantly overlaps the excitation spectrum of EYFP. The resulting energy from donor ECFP may directly excite the acceptor EYFP when the distance of two fluors is $<10 \mathrm{~nm}$ (Gordon et al., 1998). FRET measurements were performed by acceptor bleaching with a confocal laser scanning microscope (TCS SP2 AOBS; Leica, Mannheim, Germany), equipped with a $63 \times / 1.3$ numerical aperture oil immersion objective. Fluorescence for ECFP and EYFP were imaged at emission 460-490 nm (laser, $458 \mathrm{~nm}$ ) and emission 520-620 nm (laser, $514 \mathrm{~nm}$ ), respectively. In all cases, the ECFP signal was below saturation. EYFP was photobleached in the selected region of interest (ROI 1). In total, 10 neurons ( 74 neuritic granules) were traced and subjected to
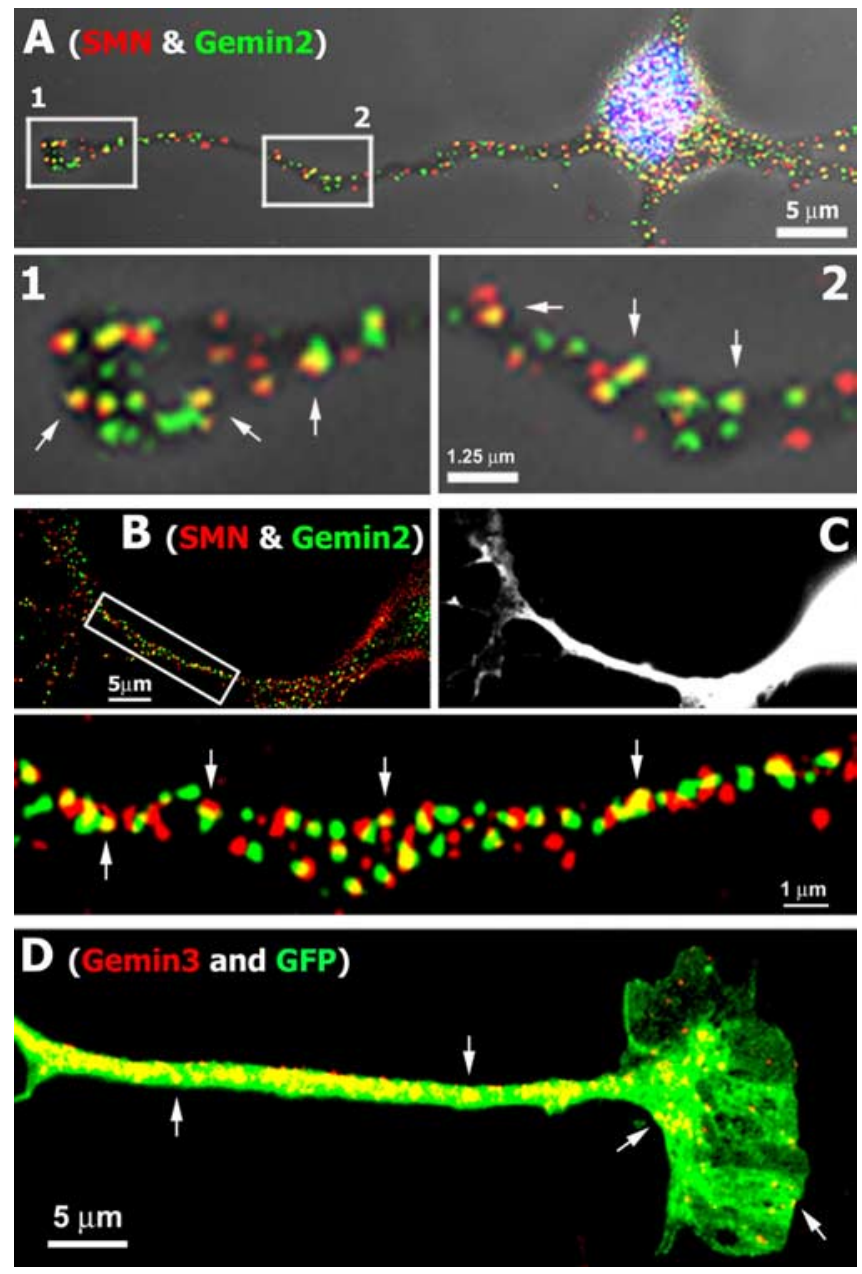

Figure 1. Colocalization of endogenous SMN and Gemin proteins in neurites and growth cones in primary forebrain culture and ES cell-derived motor neurons. A, SMN (red) and Gemin2 (green) in cultured forebrain neurons (3 DIV) were detected by double-labeled immunofluorescence using a monoclonal antibody to SMN and polyclonal antibody to Gemin2. The nucleus was stained with DAPI (blue). Higher magnification of two regions (insets 1, 2 from top panel are enlarged in bottom panel) depicts the frequent colocalization between SMN and Gemin 2 within granules in the growth cone (1, arrows) and neurite (2, arrows). $\boldsymbol{B}$, Double-labeled IF showing colocalization of SMN (red) and Gemin2 (Cy5 antibody displayed in green) in neurites of ES cell-derived differentiated motoneuron. Higher magnification of a boxed region depicts numerous granules with colocalization between SMN and Gemin2 (bottom panel, arrows). C, These cells express EGFP from a motor neuron-specific promoter. $\boldsymbol{D}$, IF detection of Gemin3 with a monoclonal antibody depicts many granules localized to the EGFP-positive axon and growth cone of the motor neuron (arrows).

FRET measurements between EYFP-SMN and ECFP-Gemin2. Six neurons coexpressing EYFP-SMN and ECFP-Gemin3 were also imaged, and 36 granules in the photobleaching area were selected for FRET measurements. ECFP fluorescence intensities within each ROI were compared before and after photobleaching. To show that FRET occurred specifically between SMN and Gemins, a small region that was outside the neurite, but within the bleached ROI, was also circled for measurements of changes in ECFP fluorescence and used as a background correction. As controls, granules were also selected from neurites that were not photobleached. FRET efficiency $\left(\mathrm{FRET}_{\mathrm{Eff}}\right)$ was calculated for each pixel from the increase of the donor fluorescence according to $\mathrm{FRET}_{\mathrm{Eff}}=$ $1-\mathrm{ECFP}_{\mathrm{Pre}} / \mathrm{ECFP}_{\text {post }}$. Percentage of the granules that showed increase of ECFP fluorescence after bleaching was defined as FRET frequency. FRET average was the mean increase of ECFP fluorescence intensities in the granules showing FRET.

To ensure that there was no bleed through between ECFP and EYFP, control transfections were done with either EYFP-SMN or ECFP-Ge- 


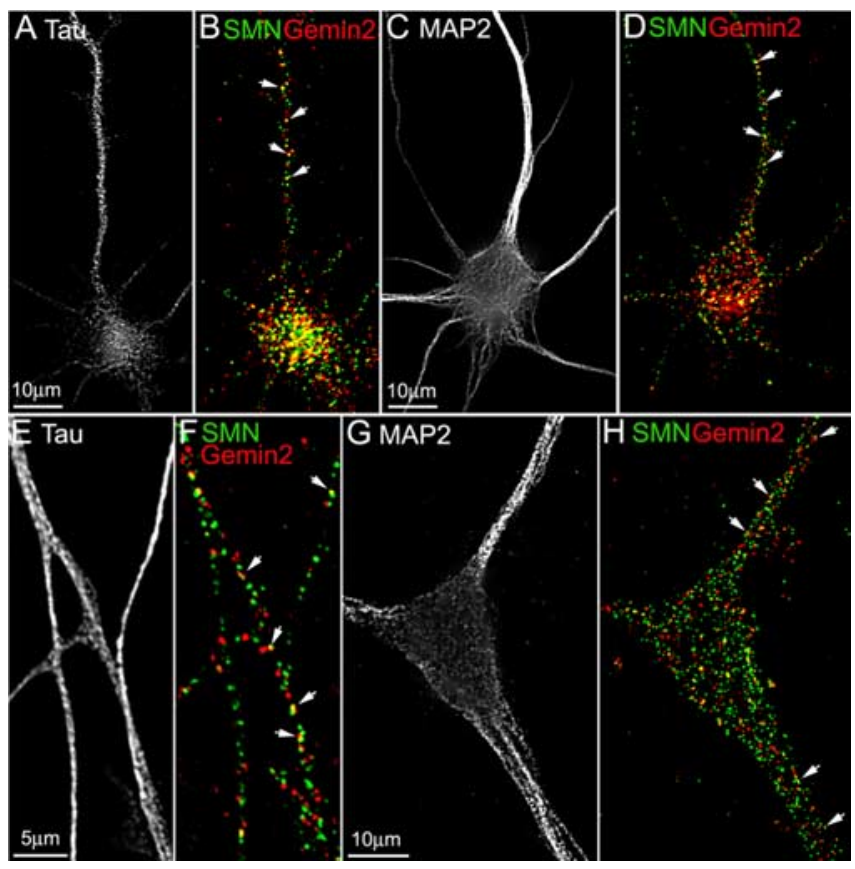

Figure 2. Colocalization of endogenous SMN and Gemin2 in axons and dendrites of differentiated cultures of primary hippocampal and motor neurons. Primary cultures of rat hippocampal neurons $(\boldsymbol{A}-\boldsymbol{D})$ and embryonic mouse motor neurons $(\boldsymbol{E}-\boldsymbol{H})$ were fixed with $4 \%$ paraformaldehyde at 7 DIV and processed for triple-labeled IF to detect the colocalization of endogenous SMN and Gemin 2 in both axons and dendrites. Monoclonal antibodies to SMN and Gemin2 were directly conjugated with Alexa Fluor 647 and Alexa Fluor 568, respectively (see Materials and Methods). Axons and dendrites were discriminated by IF staining with a polyclonal antibody to tau $(\boldsymbol{A}, \boldsymbol{E})$ or MAP2 $(\boldsymbol{C}, \boldsymbol{G})$ and $(y 2$-conjugated secondary antibody. The colocalization (indicated by arrows) of SMN (green) and Gemin2 (red) in both axons $(\boldsymbol{B}, \boldsymbol{F})$ and dendrites $(\boldsymbol{D}, \boldsymbol{H})$ of hippocampal neurons and mouse motor neurons was observed.

min2 alone, and then fluorescence intensities in the other channel was examined. In these single transfections, the ECFP or EYFP signals could only be observed and detected with their own emission.

Coimmunoprecipitation and Western blot. To show interactions of SMN with Gemin2, coimmunoprecipitation experiments were performed on transfected HEK293 cells. One day after transfection with EGFP- and Flag-tagged constructs, HEK293 cells were lysed using an ice-cold buffer (50 mm Tris- $\mathrm{HCl}, 150 \mathrm{~mm} \mathrm{NaCl}, 0.1 \% \mathrm{NP}-40$, and $0.1 \%$ sodium deoxycholate, $\mathrm{pH} 7.4)$. The total protein solution $(1 \mathrm{ml})$ was incubated with a rabbit anti-Flag antibody ( $2 \mu \mathrm{l}$; Sigma) and protein-A agarose ( $60 \mu \mathrm{l}$; Roche) at $4^{\circ} \mathrm{C}$ overnight with rotation. After washing with a high-salt buffer ( $50 \mathrm{~mm}$ Tris- $\mathrm{HCl}, 300 \mathrm{~mm} \mathrm{NaCl}$, and $0.1 \% \mathrm{NP}-40)$, the agarose pellets were resuspended in SDS-PAGE loading buffer $(60 \mu \mathrm{l})$ and heated at $100^{\circ} \mathrm{C}$ for $5 \mathrm{~min}$. Twenty microliters of the proteins from the agarose pellets were loaded onto $12 \%$ SDS-PAGE gel. The protein supernatant $(20 \mu \mathrm{l})$ from each transfection was also run onto the gel. Fractionated proteins were transferred to Hybond ECL nitrocellulose membrane (Amersham Biosciences, Arlington Heights, IL) at $4^{\circ} \mathrm{C}$ overnight. Endogenous Gemin2 or SMN was detected with monoclonal antibodies (1:2000 diluted in TBS buffer), as used for immunofluorescence staining. Monoclonal antibodies (1:4000 diluted in TBS buffer) to Flag (Sigma) or EGFP (BD Biosciences) were used for detection of the transfected fusion proteins. The membrane was washed and incubated with peroxidase-conjugated donkey anti-mouse IgG (Jackson ImmunoResearch). The signal was developed using ECL detection reagents (Amersham Biosciences).

\section{Results}

Nonrandom localization of the SMN-Gemin complex in granules within neurites and growth cones

Previous biochemical studies in non-neuronal cell lines have isolated an SMN complex with Gemin proteins and shown its func-
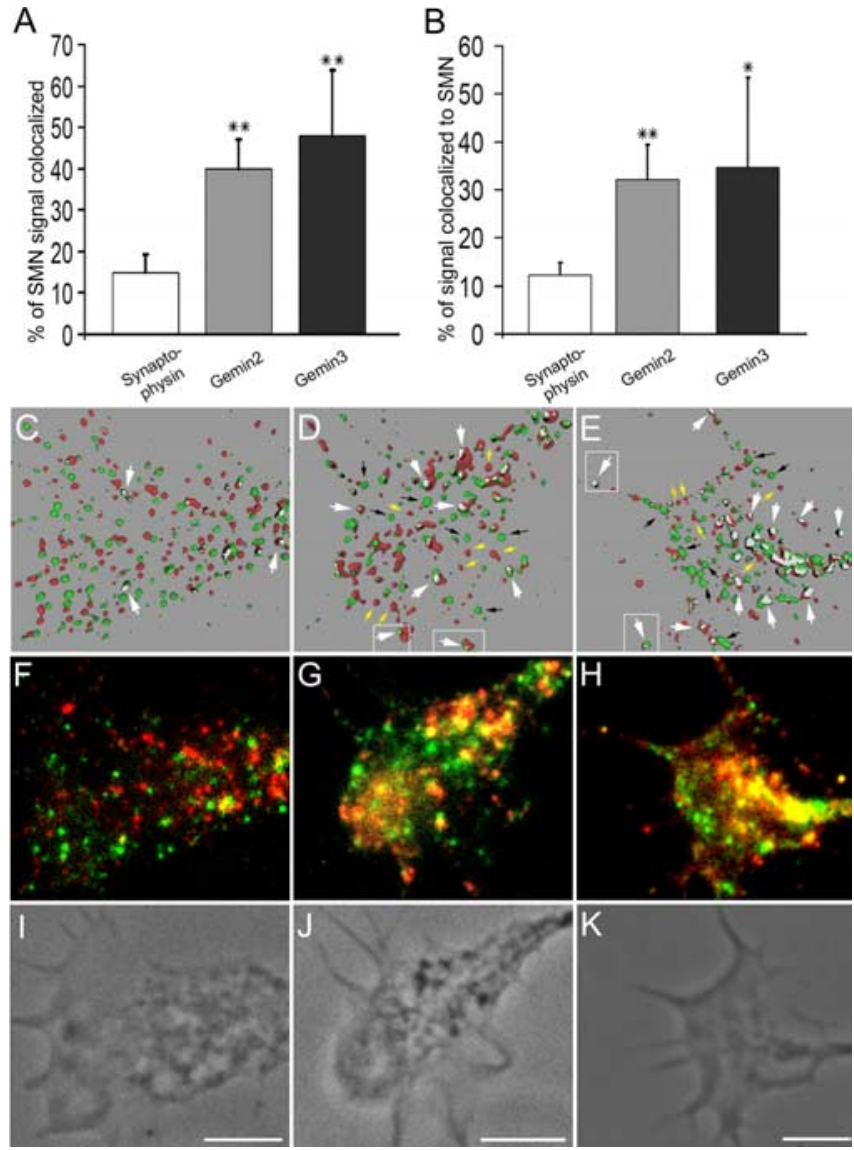

Figure 3. Quantitative analysis of SMN-Gemin colocalization in growth cones after 3D reconstruction. Hippocampal neurons were cultured for 4 DIV and processed for double-labeled immunofluorescence using directly conjugated monoclonal antibodies. Growth cones $(n=10$ per color pair) were imaged in three dimensions, deconvolved, thresholded, and statistically analyzed for percentage of voxels with colocalized signals above threshold (Imaris). $\boldsymbol{A}$, The SMN signal colocalized to Gemin2 $(40.0 \pm 7.0 \%)$ and Gemin $3(47.8 \pm 15.8 \%)$ was 1.7 and 2.2 times higher than the signal of SMN colocalized to synaptophysin $(15.0 \pm 4.5 \%)$, which was equally abundant within growth cones. $\boldsymbol{B}$, The Gemin2 signal ( $32.3 \pm 7.2 \%$ ) was 1.6 times higher than the synaptophysin signal $(12.3 \pm 2.6 \%)$ colocalized to SMN, and Gemin3 signal (34.7 \pm 18.8\%) colocalized to SMN was 1.8 times higher than synaptophysin $(12.3 \pm 2.6 \%) .{ }^{*} p<0.01$; ${ }^{* *} p<0.001$. Colocalization of SMN (green) and synaptophysin, Gemin2, or Gemin3 (red) were represented by deconvolved and reconstructed images shown in $\boldsymbol{C}, \boldsymbol{D}$, and $\boldsymbol{E}$, respectively. Colocalized voxels (white) are indicated by white arrows. Non-colocalized signals are indicated by black arrows (SMN) or yellow arrows (Gemin2 and Gemin3). The raw unprocessed data for these three growth cones are shown in $\boldsymbol{F}-\boldsymbol{H}$. Corresponding phase images are shown in $\boldsymbol{I} \boldsymbol{K}$. The presence of $S M N-G e m i n$ granules in filopodial protrusions from the growth cone is noted in boxed regions in $\boldsymbol{D}$ and $\boldsymbol{E}$. Scale bars, $5 \mu \mathrm{m}$.

tion in snRNP assembly (Gubitz et al., 2004). Here we used highresolution fluorescence imaging methods to demonstrate the formation and trafficking of SMN-Gemin multiprotein complexes in neurites and growth cones of cultured neurons. These studies were done in different types of cultured neurons: embryonic sources of primary forebrain, hippocampal and motor neurons, and ES cell-derived motor neurons. There are several past demonstrated advantages of primary forebrain and hippocampal neurons for the study of mRNA granule trafficking. These include polarization of axons and dendrites, the presence of large and flat growth cones amenable to quantitative analysis of colocalization, optimization of transfection methods to preserve growth cone morphology, and optimization of conditions for live cell imaging (Zhang et al., 2001; Tiruchinapalli et al., 2003). Here we also report the use of primary embryonic and ES cell-derived motor neurons for immunofluorescence studies. 
Double-labeled immunofluorescence on cultured forebrain neurons [ $4 \mathrm{~d}$ in vitro (DIV)] detected endogenous Gemin2 protein within granules that codistributed with SMN throughout neurites and into the growth cone (Fig. 1A). Highermagnification analysis revealed substantial colocalization of SMN (red) and Gemin2 (green) in growth cones (inset 1, arrows) and neurites (inset 2, arrows). A similar pattern of localization was observed in neurites of cultured motor neurons, derived from ES cells (Fig. $1 B$ ). SMN granules were frequently colocalized with Gemin2 (Fig. $1 B$, inset, arrows) in axons of motor neurons. These ES cell-derived motor neurons were obtained from transgenic mice that express EGFP using an HB9 promoter, specific for motor neurons (see Materials and Methods). The expression of EGFP in the same cell is shown in Figure $1 C$. Detection of Gemin 3 by immunofluorescence (red) also revealed granules that extended into the axons and growth cones of EGFP-marked motor neurons (Fig. 1D).

Primary embryonic mouse motor neurons were purified by Optiprep gradient centrifugation and cultured for $7 \mathrm{~d}$. Immunofluorescence detection of ChAT revealed positivity for this motor neuron marker in $>90 \%$ of the neurons (data not shown; see Materials and Methods).

Triple-labeled IF detection of SMN, Gemin2, and either tau or MAP2, as axonal and dendritic markers, respectively, demonstrated the presence of SMN and Gemin in both axons (Fig. $2 E, F$ ) and dendrites (Fig. 2G,H). SMN colocalization with Gemin2 was noted in many granules (arrows), yet there was also a large population of SMN and Gemin 2 granules that did not colocalize. Comparable results were also observed for SMN and Gemin localization to axons (Fig. 2A,B) and dendrites (Fig. $2 C, D)$ of hippocampal neurons cultured for 1 week.

We used primary hippocampal neurons for quantitative analysis of SMN and Gemin protein colocalization in growth cones. Hippocampal neurons, cultured on poly-lysine, often display large and flat growth cones, which were ideally suited to analyze for the possible nonrandom distribution and colocalization of SMN with Gemin proteins. Three immunofluorescence color pairs were analyzed for colocalization in individual growth cones after $3 \mathrm{D}$ reconstruction of deconvolved optical sections. Only primary antibodies were used that were directly conjugated with fluorochromes (see Materials and Methods). SMN-Gemin2 or SMN-Gemin3 were detected in the experimental group by double-labeled immunofluorescence. Using 3D colocalization analysis software (Imaris), the voxel overlap in these two datasets were compared statistically with the negative control pair (SMN-synaptophysin). A representative example is shown for SMN-synaptophysin (Fig. 3C), SMN-Ge$\min 2$ (Fig. 3D), and SMN-Gemin3 (Fig. 3E). 3D reconstructions were done on 10 growth cones from each color pair. Quantitative analyses of these $3 \mathrm{D}$ datasets demonstrated a statistically nonrandom colocalization of SMN with Gemins (Fig. 3A) or Gemins with SMN (Fig. 3B). These values ranged from a low of $33 \%$
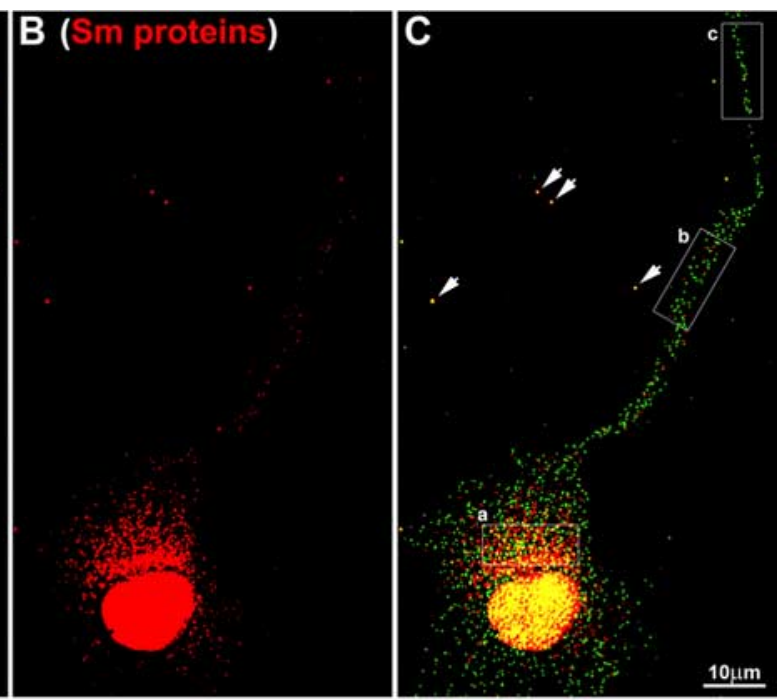

b

C
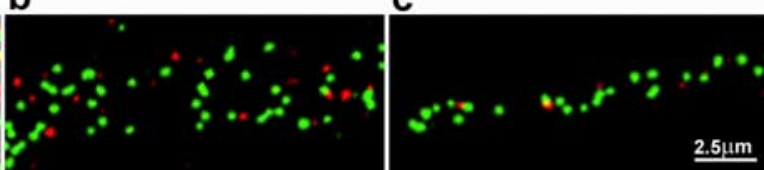

$5 \mu \mathrm{m}$

Figure 4. SMN granules within neurites are deficient of Sm proteins in motor neurons. Primary cultured mouse motor neurons , the amount of Sm proteins was extremely low with respect to SMN (enlarged inset c).

(Gemin2 with SMN) to a high of 47\% (SMN with Gemin3). In contrast, the colocalization of SMN and Gemins with synaptophysin, an equally abundant protein, only revealed at most $15 \%$ overlap. These data indicate the presence of SMN-Gemin containing granules in growth cones, yet also indicate that not all $\mathrm{SMN}$ is in a complex with Gemins and vice versa. In addition, we also noted from the growth cone analysis that SMN-Gemin granules frequently were present in filopodial protrusions (Fig. $3 D, E$, boxed regions).

In non-neuronal cells, the SMN-Gemin complex has been shown to interact with spliceosomal Sm proteins to promote the cytoplasmic assembly of snRNPs, which function in pre-mRNA splicing (Fischer et al., 1997; Yong et al., 2004). An important question addressed here is whether the SMN-Gemin granules in neurites were associated with spliceosomal Sm proteins. We hypothesized that the SMN-Gemin complex in neurites is novel and spatially distinct from those involved in snRNP assembly. Using immunofluorescence staining of primary motor neurons with a monoclonal antibody (Y12) to Sm proteins, the major component of snRNPs, we observed that Sm proteins were highly enriched in the nucleus and also showed significant staining in the perinuclear cytoplasm (Fig. $4 B$, red), in which there was colocalization with SMN (green) (Fig. 4C, inset $a$ ). SMN was also abundant in the nucleus and cytoplasm (Fig. 4A), but, in contrast to Sm proteins, SMN was prevalent throughout the neurite length (Fig. $4 C$, insets $b, c$ ). The density of Sm proteins decreased markedly with distance from the soma, in which its levels in distal neurites were very low (Fig. 4C, inset $c$ ). These results indicate that the vast majority of SMN granules in neurites lack Sm proteins, yet also indicate that $\mathrm{Sm}$ proteins may be present at very low levels. 

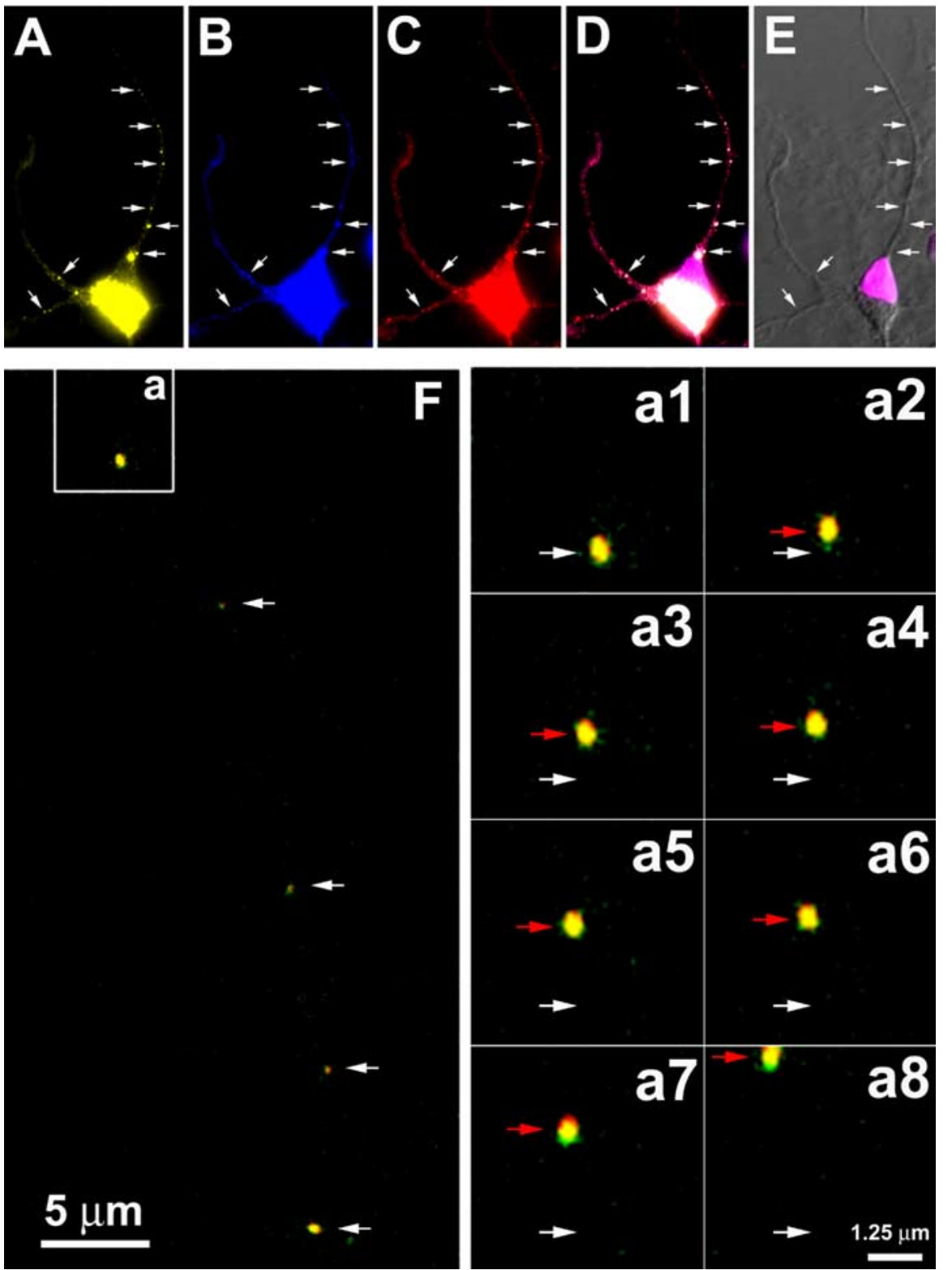

Figure 5. Colocalization and cotransport of fluorescently tagged and overexpressed SMN and Gemin2. Cultured forebrain neurons were cotransfected with EYFP-SMN ( $\boldsymbol{A}$, yellow) and ECFP-Gemin2 ( $\boldsymbol{B}$, blue) and then fixed for immunofluorescence detection of endogenous Gemin3 ( $\boldsymbol{C}$, red) using a monoclonal antibody. Colocalization of EYFP-SMN, ECFP-Gemin2, and Gemin3 within eight discrete granules is shown in neurites ( $\boldsymbol{D}$, arrows, merged signals). $\boldsymbol{E}$, The nucleus was stained with DAPI (pink) and overlaid with differential interference contrast optics. $\boldsymbol{F}$, In another experiment, neurons were cotransfected with EYFP-SMN (red) and ECFP-Gemin2 (green) and imaged in live cells using high-speed, dual-channel imaging. Five granules, containing both molecules, are depicted along the length of a neurite (arrows). One of these granules ( $\boldsymbol{a}$ ) is tracked for eight frames and displays

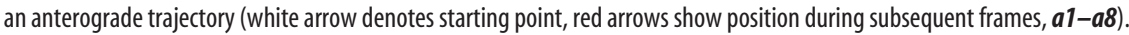

\section{Analysis of SMN-Gemin interactions using fluorescently} tagged proteins

Because SMN is known to bind Gemin2 directly in vitro (Liu et al., 1997), we used FRET analysis of fluorescently tagged and overexpressed proteins to assess direct physical interactions of SMN with Gemins in neuritic granules. This interaction and others were examined in detail using a cotransfection approach between SMN and specific Gemin proteins, using both wild-type and mutant forms tagged with different fluorescent proteins. Cultured forebrain neurons were first cotransfected with EYFPSMN and ECFP-Gemin2. All constructs were human forms of these proteins (see Materials and Methods). We first verified that the overexpressed fluorescent proteins colocalized with endogenous Gemins. Endogenous Gemin 3 was present in granules that colocalized with coexpressed EYFPSMN and ECFP-Gemin2 (Fig. 5A-E). Eight discrete granules (arrows) are depicted in two neurites showing the presence of EYFP-SMN (Fig. 5A), ECFP-Ge$\min 2$ (Fig. $5 B$ ), and endogenous Gemin3 (Fig. $5 C$ ) at the same locations (merge of all three is shown in Fig. 5D).

Live neurons expressing EYFP-SMN and ECFP-Gemin 2 were also analyzed by high-speed dual-channel imaging (see Materials and Methods). The cotransport of SMN and Gemin2 was evident within single granules that could be tracked together for several micrometers (Fig. $5 F$, white arrows in inset). Both anterograde and retrograde (data not shown) trajectories were noted.

FRET has been used to localize protein-protein interactions within cells (Jobin et al., 2003). FRET was used to quantitate molecular interactions in transfected neurons expressing EYFP-SMN and ECF$\mathrm{P}-\mathrm{Gemin} 2$, and the results from one neuron is depicted in Figure 6. Briefly, an ROI was photobleached in the EYFP (acceptor) channel (Fig. 6B,D), and then any increased fluorescence was measured in the ECFP (donor) channel. One region of interest (ROI 1) containing two neurites is shown and was analyzed before and after photobleaching (Fig. 6A-D). The fluorescence intensities within 14 encircled granules (ROI 2-ROI 15) were measured. As controls, fluorescence intensities were also examined in bleached regions outside the cell (ROI 16) and within nonbleached regions of other neurites (ROI 17-ROI 19). In total, we examined for possible FRET interactions in 74 granules from 10 transfected neurons. A high frequency (77\%) of Gemin2 granules showed increased ECFP fluorescence intensities after photobleaching when compared with before photobleaching (Fig. 6E). The average increase in ECFP fluorescence intensity (19.8\%) indicated a close interaction between EYFPSMN and ECFP-Gemin2 in single granules. In contrast, granules from nonphotobleached neurites did not show increased ECFP fluorescence, nor did we observe changes in ECFP fluorescence in photobleached regions outside the cell. Similar FRET experiments also suggested an interaction between EYFP-SMN and ECFP-Gemin3 (Fig. 6E). The observed frequency of FRET interactions between SMN-Gemin 3 was significantly lower than observed with SMN-Gemin2.

\section{Recruitment of Gemin2 into complex and granules is} dependent on interactions with SMN

We hypothesized that Gemin proteins can be recruited into SMN granules when present at stoichiometric levels and that this inter- 

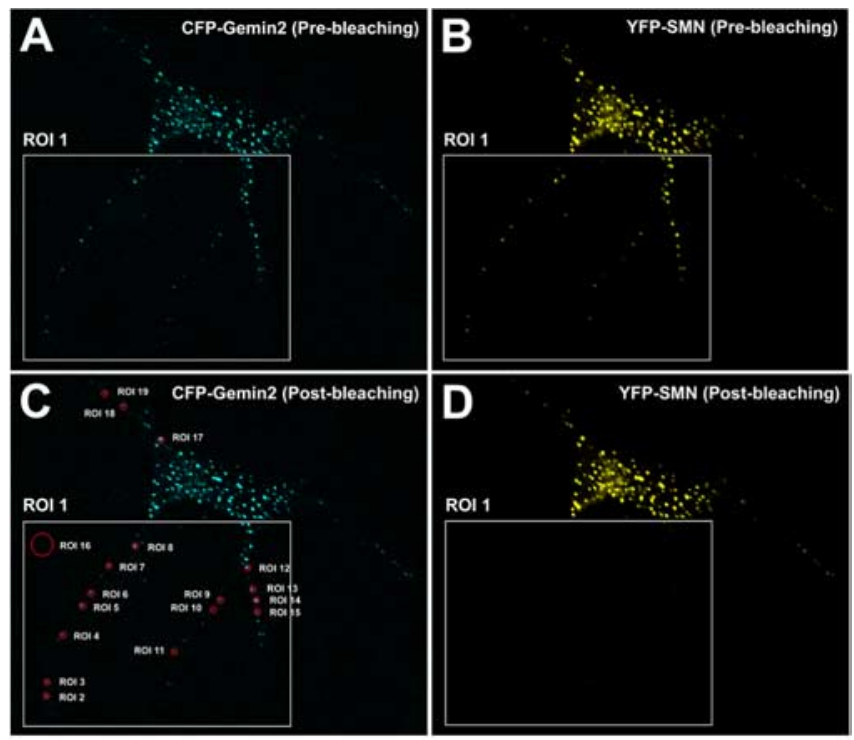

\begin{tabular}{|c|c|c|c|c|c|c|c|}
\hline \multirow[t]{2}{*}{$E$} & \multirow[b]{2}{*}{ Cells } & \multicolumn{3}{|c|}{ FRET experimental } & \multicolumn{3}{|c|}{ FRET control } \\
\hline & & Granules & $\begin{array}{l}\text { FRET } \\
\text { frequency }\end{array}$ & $\begin{array}{c}\text { FRET } \\
\text { average }\end{array}$ & Granules & $\begin{array}{l}\text { FRET } \\
\text { frequency }\end{array}$ & $\begin{array}{c}\text { FRET } \\
\text { average }\end{array}$ \\
\hline $\begin{array}{l}\text { CFP. } \\
\text { Gemin2 }\end{array}$ & 10 & 74 & $77 \%$ & $19.8 \%$ & 23 & $13 \%$ & $1.7 \%$ \\
\hline $\begin{array}{l}\text { CFP. } \\
\text { Gemin3 }\end{array}$ & 6 & 36 & $56 \%$ & $19.6 \%$ & 6 & $17 \%$ & $3.0 \%$ \\
\hline
\end{tabular}

Figure 6. FRET analysis depicts interaction between EYFP-SMN and ECFP-Gemin2 or ECFPGemin3 in neurites. FRET was used to detect protein-protein interactions between SMN and Gemins in neurites. Cultured forebrain neurons were transfected with ECFP-Gemin2 or ECFPGemin3 as the donor and EYFP-SMN as the acceptor fluorophore, for FRET analysis. Fluorescence was imaged using a confocal microscope (see Materials and Methods). One neuron is shown here as an example. $\boldsymbol{A}, \boldsymbol{B}$, ECFP-Gemin2 granules ( $\boldsymbol{A}$, blue) and EYFP-SMN granules ( $\boldsymbol{B}$, yellow) before photobleaching of EYFP. C, ECFP-Gemin2 fluorescence after photobleaching of boxed region (ROI 1). Each granule within ROI 1 was outlined in red and indicated as ROI2-ROI 15. An area (ROI 16) outside the neurite was also circled within ROI 1 as a control for measurement of increased fluorescence in ECFP channel after photobleaching of EYFP. Three granules, labeled as ROl 17-ROI 19, in another neurite that was not photobleached, were used as additional controls for FRET measurements. D, Fluorescence signals of EYFP-SMN granules were eliminated by photobleaching with a laser in the selected area (ROI 1). $\boldsymbol{E}$, FRET measurements are indicated in the table. FRET frequency indicates the percentage of granules showing increased ECFP fluorescence after photobleaching of EYFP. FRET average indicates the percentage increase in ECFP fluorescence after EYFP photobleaching.

action is dependent on specific domain(s) of SMN. After EGFPGemin 2 overexpression (green) by itself, neurons were fixed for IF analysis of endogenous SMN (red), and the nucleus was stained with 4',6'-diamidino-2-phenylindole (DAPI) (blue). The EGFP-Gemin2 fluorescence was diffuse and filled nucleus, cytoplasm, and processes (Fig. 7A). This finding suggests that the endogenous SMN within neuritic granules is in a complex with the endogenous Gemins or other proteins and is unavailable to bind to the overexpressed Gemin2. Consistent with this idea, when EGFP-Gemin2 was cotransfected with Flag-SMN (red), the EGFP-Gemin 2 signal was now granular and colocalized with SMN (red); both proteins were often coenriched in neuritic filopodia and growth cones (Fig. $7 B$, inset arrows).

We then investigated whether deletion of the SMN N terminus, which contains the Gemin2 binding site (Liu et al., 1997; Wang and Dreyfuss, 2001b), was required for the recruitment of Gemin 2 into granules by SMN after their cotransfection. First, we show here that single expression of ECFP-Gemin2 (Fig. 8A) or ECFP-Gemin3 (Fig. 8D) was diffuse and nongranular, whereas coexpression of ECFP-Gemin2 or ECFP-Gemin3 with EYFP$\mathrm{SMN}$ showed their colocalization in granules (Fig. $8 \mathrm{~B}, \mathrm{E}$, arrows). An N-terminal deletion mutant of SMN (EYFP-SMN $\Delta$ N53) was unable to recruit the overexpressed ECFP-Gemin2 into granules (Fig. 8C), whereas EYFP-SMN $\Delta$ N53 was still able to form neuritic granules (Fig. $8 C$, arrows), and ECFP-Gemin2 (green) was diffuse through the cytoplasm and nucleus. Deletion of the $\mathrm{N}$ terminus of EYFP-SMN did not affect colocalization with ECFPGemin3 in neuritic granules (Fig. $8 F$, arrows). These data show that the colocalization of SMN and Gemin 2 within neuritic granules is dependent on their levels in the cell and known molecular interactions. In addition, these results show that the $\mathrm{N}$ terminus of SMN is not necessary for granule formation or interactions with Gemin3.

co-IP and Western blot experiments in transfected HEK293 cells were used to show that Gemin 2 was stabilized by its interaction with SMN. First, we show that Gemin2 could be coprecipitated with both Flag-SMN and Flag-SMN $\Delta 7$ (Fig. 8G, lanes 1, 5) but not with Flag-SMN $\Delta$ N53 (Fig. 8G, lane 3), which lacks the Gemin 2 binding site. EGFP-Gemin 2 was also only weakly apparent in the protein supernatant from cells expressing FlagSMN $\Delta$ N53 (Fig. 8G, lane 4). These low levels of EGFP-Gemin2 in both pellets and supernatants from cells expressing FlagSMN $\Delta$ N53 compared with cells expressing Flag-SMN and FlagSMN $\Delta 7$ suggested that EGFP-Gemin 2 could be stabilized by interactions with the $\mathrm{N}$ terminus of SMN. This idea was further supported by Western blot analysis. EGFP-Gemin2 levels were very low when it was expressed by itself (Fig. $8 H$, lanes $1-3$ ) when compared with cotransfection with full-length SMN (Fig. $8 \mathrm{H}$, lanes 4-6). For example, at the $12 \mathrm{~h}$ time point, EGFP-Gemin 2 is barely apparent on the gel (Fig. $8 \mathrm{H}$, lane 1 ), whereas a distinct band is noted when coexpressed with full-length SMN (Fig. $8 \mathrm{H}$, lane 4). EGFP-Gemin 2 and Flag-SMN levels were both substantially increased over the $48 \mathrm{~h}$ time course. In contrast, EGFPGemin2 levels were consistently lower in cells cotransfected with Flag-SMN $\Delta$ N53 (Fig. $8 H$, lanes 7-9). These results are consistent with previous in vitro observations that Gemin2 is unstable when not bound with SMN (Wang et al., 2001). Collectively, our findings suggest that a stable SMN-Gemin complex exists in granules in neuronal processes and that the molecular interactions between SMN and Gemins are required for maintaining Gemin proteins in this stable complex.

\section{Discussion}

\section{Localization of an SMN-Gemin complex in axons and} dendrites that is deficient of spliceosomal Sm proteins

Previous immunocytochemical studies have documented the localization of the SMN protein to axons and dendrites of spinal cord neurons in vivo (Battaglia et al., 1997; Bechade et al., 1999; Pagliardini et al., 2000). In cultured forebrain neurons, we have shown previously that SMN is localized in granules that are actively transported into developing neurites and growth cones (Zhang et al., 2003). In an effort toward understanding the function of SMN in neuronal processes and how loss of SMN leads to SMA, it is essential to identify proteins that associate with SMN in neuronal processes. Ideally, these studies should be conducted in motor neurons.

Because previous biochemistry studies in immortalized nonneuronal cells have shown that SMN is present in a tight complex with several Gemin proteins (Paushkin et al., 2002), it is critical to know whether the SMN-Gemin complex is present in neuronal processes and growth cones. Previous studies have had conflicting results on whether these proteins colocalize in neuronal pro- 

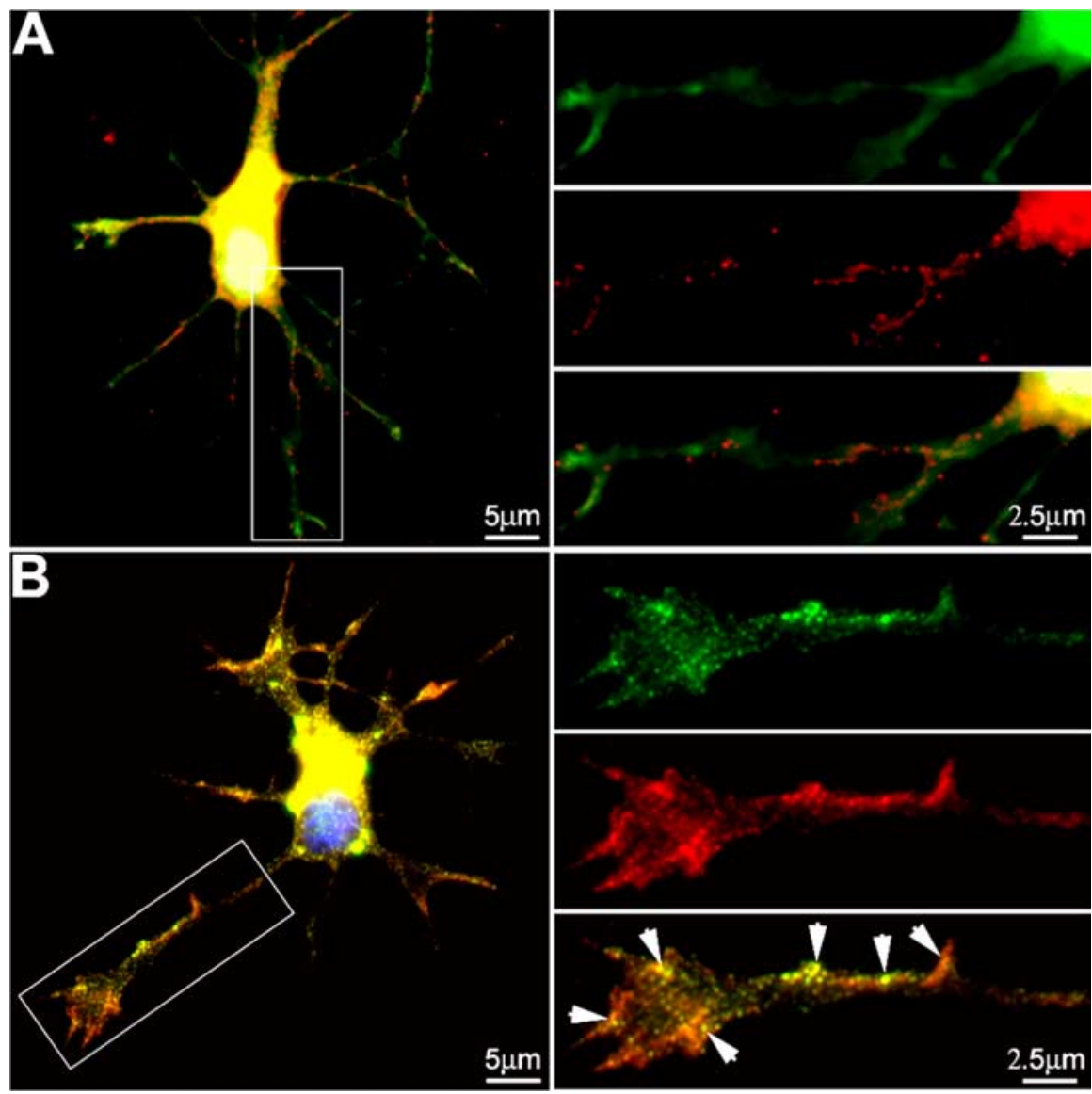

Figure 7. Gemin2 is recruited by SMN into granules within neurites and growth cones. $\boldsymbol{A}$, After transfection of EGFP-Gemin2 (green), cultured forebrain neurons (4 DIV) were fixed for IF analysis of endogenous SMN (red), and the nucleus was stained with DAPI (blue). The EGFP-Gemin2 fluorescence was mostly diffuse and filled all cellular regions including cytoplasmic processes and the nucleus. Note that the blue stain of the nucleus was not visible in the merged overlay because of the strong EGFP signal. The distribution of SMN (red) was highly granular or punctate as described previously (Zhang et al., 2003). One neurite from the boxed inset is enlarged at right to show EGFP-Gemin2 (top), SMN (middle), and overlay (bottom). $\boldsymbol{B}$, In contrast, when EGFP-Gemin2 was cotransfected with Flag-SMN (red), the EGFP-Gemin2 signal was then granular and frequently colocalized with SMN (red) in neurites, growth cones, and filopodia (arrows). One neurite (boxed inset) is enlarged to the right and shows EGFP-Gemin2 (top), Flag-SMN (middle), and overlay (bottom). Note also that the DAPI-stained nucleus (blue) is now visible, because the EGFPGemin2 signal is observed in puncta (likely gems) in contrast to filling the entire nucleus as in $\boldsymbol{A}$.

cesses (Jablonka et al., 2001; Sharma et al., 2005). One confounding factor is that only conventional immunofluorescence analysis was performed in these previous studies. Although this method may be useful to show that both proteins are present in a neurite or growth cone, it is not possible to know whether they associate together in single particles or granules. This type of analysis necessitated use of higher-resolution imaging methods. In this study, we demonstrate, for the first time, the colocalization of SMN and Gemin2 and Gemin3 in granules that distribute to neuronal processes and growth cones of primary hippocampal and motor neurons, and ES cell-derived motor neurons. Quantitative analyses of $3 \mathrm{D}$ reconstructed growth cones indicate a statistically nonrandom association of SMN with Gemin2 (40\%) and Gemin3 (48\%). In addition, we used fluorescently tagged and overexpressed SMN and Gemin proteins to demonstrate an FRET interaction and cotransport of complex. Our data also suggest the presence of SMN and Gemin particles that do not colocalize. These results suggest the presence of diverse SMNcontaining multiprotein complexes in neuronal processes. Our observations for the lack of spliceosomal Sm proteins in processes suggests an additional function for SMN complexes that are lo- calized to neuronal processes. We speculate that the SMN-Gemin complex may play a role in some aspect of mRNP assembly, as it has been shown for snRNP assembly (Briese et al., 2005; Monani, 2005).

\section{Possible functions for SMN} multiprotein complexes in neurons? One possible function for the SMN-Gemin complex may be the assembly of a localized $\beta$-actin mRNP complex. A seminal report by Rossoll et al. (2003) has shown that motor neurons cultured from an SMA transgenic mouse model, having low SMN levels, showed reduced localization of $\beta$-actin mRNA and protein in axonal growth cones. Axons were also shorter in length and their growth cones were smaller in size; there was no evidence for dendritic impairments (Rossoll et al., 2003). These findings provide evidence of a role for SMN in some aspect of the well studied molecular mechanism of $\beta$-actin mRNA localization and its function in $\beta$-actin protein sorting and axon growth cone motility (Zhang et al., 1999, 2001). Because the assembly of a $\beta$-actin mRNA localization complex appears to involve the coordinated binding of two mRNA binding proteins, Z-DNA binding protein 1 (ZBP1) and ZBP2, to a stem-loop structure within a $54 \mathrm{nt}$ zip-code sequence within the $3^{\prime}$ untranslated region (Ross et al., 1997; Gu et al., 2002), we speculate that the SMN-Gemin complex might facilitate these molecular interactions. In addition, it has been shown that SMN interacts with the mRNA binding protein hnRNP-R, which colocalized with SMN in processes of motor neurons (Rossoll et al., 2002). Moreover, hnRNP-R associates with $\beta$-actin mRNA in vitro and enhances its localization in neurites when overexpressed in PC12 cells (Rossoll et al., 2003). It will be of interest to know whether ZBP1 interacts with hnRNP-R and whether the SMN-Gemin complex may facilitate these interactions to affect assembly of a $\beta$-actin mRNP complex that is then localized.

Because mRNA localization and translation in growth cones have been linked to growth cone motility and axon guidance (Martin, 2004), it will be interesting to also assess a possible function for the SMN-Gemin complex in translational regulation during axonal pathfinding. Our data indicate that the SMN-Gemin complex is frequently present within lamellar and filopodial protrusions from axonal growth cones. An exciting report has shown that knockdown of SMN in zebrafish resulted in axonspecific pathfinding defects in motor neurons, which were partially rescued by full-length human SMN but not by SMN $\Delta 7$ (McWhorter et al., 2003). As future studies may reveal a role for $\beta$-actin mRNA localization and its local translation in axon guidance, it will be important to examine how an SMN complex may be involved in this process.

Because SMN is also localized to dendrites of adult motor neurons in vivo (Bechade et al., 1999) and in developing dendrites 
in culture at 7 DIV (Fig. 2), additional work is needed to assess a possible functional role for $\mathrm{SMN}$ in dendrites. Although the study by Rossoll et al. (2003) did not observe gross defects in dendritic development or branching in cultured SMA motor neurons, it is possible that SMN may play a role in dendrites at the synapse. In addition to $\beta$-actin mRNA localization to the developing axon (Zhang et al., 2001), we later showed that it is also localized to developing dendrites (Eom et al., 2003). ZBP1-mediated localization of $\beta$-actin mRNA was shown to be necessary for the formation of filopodia and filopodial synapses in developing dendrites in response to BDNF stimulation (Eom et al., 2003). Perhaps SMN may interact with this dendritic ZBP1- $\beta$-actin mRNP complex. Another possible dendritic interaction for $\mathrm{SMN}$ is suggested by the findings that the SMN-interacting protein SYNCRIP (synaptotagmin binding, cytoplasmic RNA interacting protein) (hnRNP-Q1) (Rossoll et al., 2002) is cotransported in dendritic RNA granules with the inositol triphosphate receptor mRNAs (Bannai et al., 2004).

SMN may also function in other less well defined aspects of ribonucleoprotein regulation in the cytoplasm. An interesting report has shown that SMN colocalized with markers for stress granules (SGs), i.e., TIA-1/R and G3BP, and could induce their formation during overexpression (Hua and Zhou, 2004). Stress granules serve as depots to recruit poly(A) mRNA, poly(A)binding protein, ribosomes, and translational components in response to environmental stresses, such as heat shock and oxidative stress (Kedersha et al., 1999). mRNAs within SGs are translationally repressed, and, after recovery from the stress response, they are released from SGs and translated. A role for SMN in SG formation may have important implications for understanding the molecular basis for axon degeneration in SMA.

\section{Molecular interactions for assembly and} localization of SMN-Gemin complexes An N-terminal deletion of SMN (SMN $\Delta 53)$, which cannot bind Gemin2 (Wang and Dreyfuss, 2001b), was able to form granules and be transported into processes. Gemin 2 was not recruited into SMN $\Delta 53$ granules and was diffusely distributed. These data show that molecular interactions known to be involved in SMN binding to Gemin2 are necessary for the recruitment of Gemin 2 into SMN granules. However, SMN can indeed form granules and be transported in the absence of interactions with Gemin2. We also show that overexpression of either Gemin 2 or Gemin 3 individually (without SMN) was diffuse and nongranular, likely because their levels were in excess of the en-
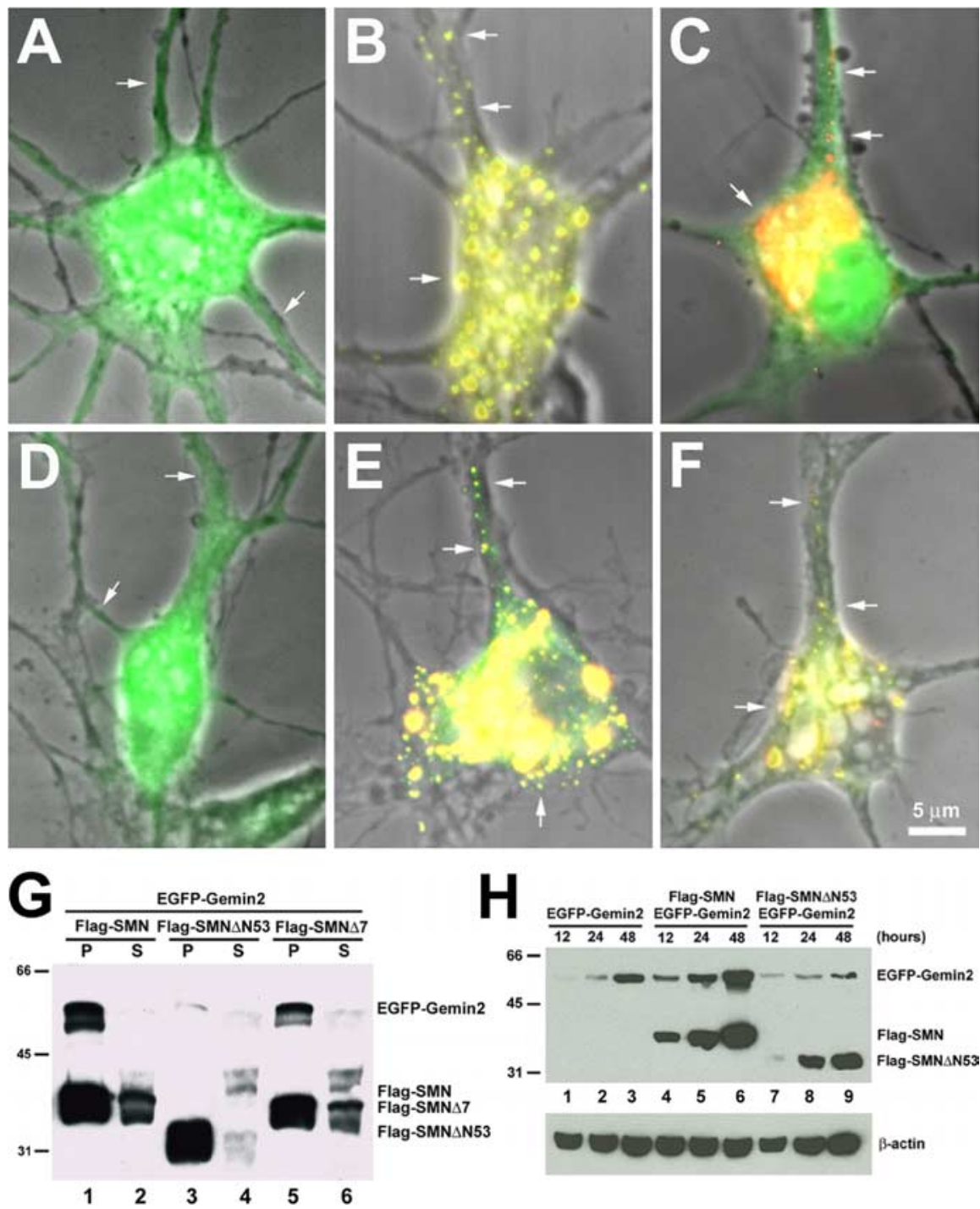

Figure 8. Interactions between Gemin2 with SMN necessary for recruitment into granules and to stabilize Gemin2. $\boldsymbol{A}, \boldsymbol{D}$, Overexpression of ECFP-Gemin2 (A) or ECFP-Gemin3 (D) by themselves in cultured forebrain neurons (4 DIV) showed diffuse and uniform fluorescence, with no evidence for discrete granules (green, arrows). $\boldsymbol{B}, \boldsymbol{E}$, In contrast, when ECFP-Gemin2 ( $\boldsymbol{B}$, green) or ECFP-Gemin3 ( $\boldsymbol{E}$, green) were cotransfected with EYFP-SMN (red), the recruitment of Gemins into SMN granules was observed ( $\boldsymbol{B}, \boldsymbol{E}$, merged images, arrows). $\boldsymbol{C}$, In contrast, coexpression of the N-terminal deletion mutant of SMN (EYFP-SMN $\Delta$ N53), which lacks the known binding site for Gemin2, was not able to recruit Gemin2 into granules. EYFP-SMN $\Delta$ N53 (red) showed a granular pattern in neurites ( $\boldsymbol{C}$, arrows), yet ECFP-Gemin2 ( $\boldsymbol{C}$, green) remained in a diffuse pattern. The red and green signal in the neurite showed little colocalization. $\boldsymbol{F}$, ECFP-Gemin3 (green) was recruited into granules by cotransfection with EYFP-SMN $\Delta$ N53 (red), as evident by colocalization of the merged images (yellow, arrows). G, Gemin2 stabilization by interactions with SMN. HEK293 cells were cotransfected with EGFP-Gemin2 and Flag-tagged SMN constructs. P, Pellet; S, Supernatant. Cells were lysed, immunoprecipitated with anti-Flag antibodies, and analyzed for EGFP and Flag expression by Western blot. EGFP-Gemin2 was coimmunoprecipitated with Flag-SMN (lane 1) or Flag-SMN $\Delta 7$ (lane 5) but not Flag-SMN $\Delta$ N53 (lane 3), which lacks the known Gemin 2 binding domain. $\boldsymbol{H}$, Western blot analysis at 12,24 , and $48 \mathrm{~h}$ expression showed that EGFP-Gemin 2 was present at higher levels over time when coexpressed with Flag-SMN (lanes 4-6) compared with when Gemin2 was expressed by itself (lanes 1-3) or coexpressed with Flag-SMN $\Delta$ N53 (lanes 7-9). Flag- and EGFP-tagged proteins were detected with monoclonal antibodies. $\beta$-Actin was detected with a monoclonal antibody as loading control.

dogenous SMN and insufficient to be recruited into granules. These findings indicate that the stoichiometry between SMN and Gemin proteins are important factors that influence assembly of proteins into granules.

The association of Gemin2 with SMN was also shown to stabilize Gemin2. Western blot analysis of cells cotransfected with Gemin2 and SMN led to increased Gemin2 levels over time after transfection. Collectively, our results suggest that the binding of Gemin2 to the $\mathrm{N}$ terminus of SMN is required for Gemin2 to 
form granules, and this biochemical association within granules has a stabilizing effect on Gemin2. Previous biochemical studies have also provided evidence for the stabilization of Gemin2 by SMN (Jablonka et al., 2001; Wang and Dreyfuss, 2001a). There has been genetic evidence suggesting a functional interaction between SMN and Gemin2. Gemin2/Smn double-heterozygous deficient mice showed motor neuron degeneration that was significantly higher than in Smn heterozygous mice (Jablonka et al., 2002).

\section{Summary}

In this study, high-resolution fluorescence and quantitative imaging methods were used to document the presence and cotransport of an SMN-Gemin complex in neuronal processes and growth cones of primary motor and hippocampal neurons and ES cell-derived motor neurons. In contrast, spliceosomal Sm proteins were confined to the nucleus and perinuclear cytoplasm. In addition, our data suggest the presence of SMN and Gemin containing particles that do not colocalize. These results suggest the presence of diverse types of SMN complexes that have functions in neurons, other than its well characterized role to assemble snRNPs in all cells. Future efforts to identify specific RNPs in axons and dendrites that are regulated by these SMN complexes will be critical toward understanding the molecular basis for spinal muscular atrophy. An attractive hypothesis is that the SMNGemin complex plays a role in the assembly of localized mRNP complexes needed for axonal growth and/or synaptogenesis. The dying-back axonopathy and degeneration of motor neurons in SMA models (Nicole et al., 2002) may be attributed, in part, to deficiencies in the regulated localization and translation of mRNA during neuronal development. Regarding efforts for therapies in SMA, ES cell-derived motor neurons, which also were shown to localize SMN complexes, could be used to address whether pharmacologic manipulation can affect functional levels of RNP complexes in neuronal processes.

\section{References}

Antar L, Afroz R, Dictenberg J, Carroll R, Bassell G (2004) Metabotropic glutamate receptor activation regulates fragile $\mathrm{x}$ mental retardation protein and FMR1 mRNA localization differentially in dendrites and at synapses. J Neurosci 24:2648-2655.

Arce V, Garces A, de Bovis B, Filippi P, Henderson C, Pettmann B, deLapeyriere O (1999) Cardiotrophin-1 requires LIFRbeta to promote survival of mouse motoneurons purified by a novel technique. J Neurosci Res 55:119-126.

Bannai H, Fukatsu K, Mizutani A, Natsume T, Iemura S, Ikegami T, Inoue T, Mikoshiba K (2004) An RNA-interacting protein, SYNCRIP (heterogeneous nuclear ribonuclear protein Q1/NSAP1) is a component of mRNA granule transported with inositol 1,4,5-trisphosphate receptor type 1 mRNA in neuronal dendrites. J Biol Chem 279:53427-53434.

Battaglia G, Princivalle A, Forti F, Lizier C, Zeviani M (1997) Expression of the SMN gene, the spinal muscular atrophy determining gene, in the mammalian central nervous system. Hum Mol Genet 6:1961-1971.

Bechade C, Rostaing P, Cisterni C, Kalisch R, La Bella V, Pettmann B, Triller A (1999) Subcellular distribution of survival motor neuron (SMN) protein: possible involvement in nucleocytoplasmic and dendritic transport. Eur J Neurosci 11:293-304.

Briese M, Esmaeili B, Sattelle DB (2005) Is spinal muscular atrophy the result of defects in motor neuron processes? BioEssays 27:946-957.

Campbell DS, Holt CE (2001) Chemotropic responses of retinal growth cones mediated by rapid local protein synthesis and degradation. Neuron 32:1013-1026.

Carvalho T, Almeida F, Calapez A, Lafarga M, Berciano MT, Carmo-Fonseca M (1999) The spinal muscular atrophy disease gene product, SMN: a link between snRNP biogenesis and the Cajal (coiled) body. J Cell Biol 147:715-728.
Eom T, Antar LN, Singer RH, Bassell GJ (2003) Localization of a $\beta$-actin mRNP complex with zipcode-binding protein modulates the density of dendritic filopodia and filopodial synapses. J. Neurosci 23:1043310444.

Fischer U, Liu Q, Dreyfuss G (1997) The SMN-SIP1 complex has an essential role in spliceosomal snRNP biogenesis. Cell 90:1023-1029.

Frank-Kamenetsky M, Zhang XM, Bottega S, Guicherit O, Wichterle H, Dudek H, Bumcrot D, Wang FY, Jones S, Shulok J, , (2002) Smallmolecule modulators of Hedgehog signaling: identification and characterization of Smoothened agonists and antagonists. J Biol 1:10.

Frugier T, Nicole S, Cifuentes-Diaz C, Melki J (2002) The molecular bases of spinal muscular atrophy. Curr Opin Genet Dev 12:294-298.

Gordon GW, Berry G, Liang XH, Levine B, Herman B (1998) Quantitative fluorescence resonance energy transfer measurements using fluorescence microscopy. Biophys J 74:2702-2713.

Gu W, Pan F, Zhang HL, Bassell CJ, Singer RH (2002) A predominantly nuclear protein affecting cytoplasmic localization of $\beta$-actin mRNA in fibroblasts and neurons. J Cell Biol 156:41-51.

Gubitz AK, Feng W, Dreyfuss G (2004) The SMN complex. Exp Cell Res 296:51-56.

Hua Y, Zhou J (2004) Survival motor neuron protein facilitates assembly of stress granules. FEBS Lett 572:69-74.

Jablonka S, Bandilla M, Wiese S, Buhler D, Wirth B, Sendtner M, Fischer U (2001) Co-regulation of survival of motor neuron (SMN) protein and its interactor SIP1 during development and in spinal muscular atrophy. Hum Mol Genet 10:497-505.

Jablonka S, Holtmann B, Meister G, Bandilla M, Rossoll W, Fischer U, Sendtner M (2002) Gene targeting of Gemin2 in mice reveals a correlation between defects in the biogenesis of $U$ snRNPs and motoneuron cell death. Proc Natl Acad Sci USA 99:10126-10131.

Jobin CM, Chen H, Lin AJ, Yacono PW, Igarashi J, Michel T, Golan DE (2003) Receptor-regulated dynamic interaction between endothelial nitric oxide synthase and calmodulin revealed by fluorescence resonance energy transfer in living cells. Biochemistry 42:11716-11725.

Kedersha NL, Gupta M, Li W, Miller I, Anderson P (1999) RNAbinding proteins TIA-1 and TIAR link the phosphorylation of eIF-2 alpha to the assembly of mammalian stress granules. J Cell Biol 147:1431-1442.

Liu Q, Dreyfuss G (1996) A novel nuclear structure containing the survival of motor neurons protein. EMBO J 15:3555-3565.

Liu Q, Fischer U, Wang F, Dreyfuss G (1997) The spinal muscular atrophy disease gene product, SMN, and its associated protein SIP1 are in a complex with spliceosomal snRNP proteins. Cell 90:1013-1021.

Martin KC (2004) Local protein synthesis during axon guidance and synaptic plasticity. Curr Opin Neurobiol 14:305-310.

McWhorter ML, Monani UR, Burghes AH, Beattie CE (2003) Knockdown of the survival motor neuron (Smn) protein in zebrafish causes defects in motor axon outgrowth and pathfinding. J Cell Biol 162:919-931.

Monani UR (2005) Spinal muscular atrophy: a deficiency in a ubiquitous protein; a motor neuron-specific disease. Neuron 48:885-896.

Monani UR, Sendtner M, Coovert DD, Rossol W, Morris GE, Burghes AH (2000) The human SMN2 gene rescues embryonic lethality in $\mathrm{SMN}^{-1-}$ mice and results in a mouse with SMA. Hum Mol Genet 9:333-339.

Nicole S, Diaz CC, Frugier T, Melki J (2002) Spinal muscular atrophy: recent advances and future prospects. Muscle Nerve 26:4-13.

Pagliardini S, Giavazzi A, Setola V, Lizier C, Di Luca M, DeBiasi S, Battaglia G (2000) Subcellular localization and axonal transport of the survival motor neuron (SMN) protein in the developing rat spinal cord. Hum Mol Genet 9:47-56.

Paushkin S, Gubitz AK, Massenet S, Dreyfuss G (2002) The SMN complex, an assemblyosome of ribonucleoproteins. Curr Opin Cell Biol 14:305-312.

Ross A, Oleynikov Y, Kislauskis E, Taneja K, Singer R (1997) Characterization of a $\beta$-actin mRNA zipcode-binding protein. Mol Cell Biol 17:2158-2165.

Rossoll W, Kroning AK, Ohndorf UM, Steegborn C, Jablonka S, Sendtner M (2002) Specific interaction of Smn, the spinal muscular atrophy determining gene product, with hnRNP-R and gry-rbp/hnRNP-Q: a role for Smn in RNA processing in motor axons? Hum Mol Genet 11:93-105.

Rossoll W, Jablonka S, Andreassi C, Kroning AK, Karle K, Monani UR, Send- 
tner M (2003) Smn, the spinal muscular atrophy-determining gene product, modulates axon growth and localization of $\beta$-actin mRNA in growth cones of motoneurons. J Cell Biol 163:801-812.

Sharma A, Lambrechts A, Hao le T, Le TT, Sewry CA, Ampe C, Burghes AH, Morris GE (2005) A role for complexes of survival of motor neurons $(\mathrm{SMN})$ protein with gemins and profilin in neurite-like cytoplasmic extensions of cultured nerve cells. Exp Cell Res 309:185-197.

Tiruchinapalli DM, Oleynikov Y, Kelic S, Shenoy SM, Hartley A, Stanton PK, Singer RH, Bassell GJ (2003) Activity-dependent trafficking and dynamic localization of zipcode binding protein 1 and $\beta$-actin mRNA in dendrites and spines of hippocampal neurons. J Neurosci 23:32513261.

Wang J, Dreyfuss G (2001a) A cell system with targeted disruption of the SMN gene: functional conservation of the SMN protein and dependence of Gemin2 on SMN. J Biol Chem 276:9599-9605.

Wang J, Dreyfuss G (2001b) Characterization of functional domains of the SMN protein in vivo. J Biol Chem 276:45387-45393.
Wichterle H, Lieberam I, Porter J, Jessell T (2002) Directed differentiation of embryonic stem cells into motor neurons. Cell 110:385-397.

Yong J, Wan L, Dreyfuss G (2004) Why do cells need an assembly machine for RNA-protein complexes? Trends Cell Biol 14:226-232.

Young PJ, Le TT, Nguyen TM, Burghes AH, Morris GE (2000) The relationship between SMN, the spinal muscular atrophy protein, and nuclear colied bodies in differentiated tissues and cultured cells. Exp Cell Res 256:365-374.

Zhang HL, Singer RH, Bassell GJ (1999) Neurotrophin regulation of betaactin mRNA and protein localization within growth cones. J Cell Biol 147:59-70.

Zhang HL, Eom T, Oleynikov Y, Shenoy SM, Lieblet DA, Dictenberg JB, Singer RH, Bassell GJ (2001) Neurotrophin induced transport of $\beta$-actin mRNP complex increases $\beta$-actin levels and stimulates growth cone motility. Neuron 31:261-275.

Zhang HL, Pan F, Hong D, Shenoy SM, Singer RH, Bassell GJ (2003) Active transport of the survival motor neuron protein and the role of exon-7 in cytoplasmic localization. J Neurosci 23:6627-6637. 